\title{
Global Existence and Finite Time Blow-up of Solutions to a Nonlocal $p$-Laplace Equation
}

\author{
Jian $\mathrm{Li}^{a}$ and Yuzhu $\mathrm{Han}^{b}$ \\ ${ }^{a}$ College of Information Technology, Jilin Agricultural University \\ No. 2888, Xincheng St, Changchun 130118, China \\ ${ }^{b}$ School of Mathematics, Jilin University \\ No. 2699, Qianjin St, Changchun 130012, China \\ E-mail(corresp.): yzhan@jlu.edu.cn \\ E-mail: liemperor@163.com
}

Received June 20, 2018; revised January 11, 2019; accepted January 11, 2019

\begin{abstract}
In this paper a class of nonlocal diffusion equations associated with a $p$-Laplace operator, usually referred to as $p$-Kirchhoff equations, are studied. By applying Galerkin's approximation and the modified potential well method, we obtain a threshold result for the solutions to exist globally or to blow up in finite time for subcritical and critical initial energy. The decay rate of the $L^{2}$ norm is also obtained for global solutions. When the initial energy is supercritical, an abstract criterion is given for the solutions to exist globally or to blow up in finite time, in terms of two variational numbers. These generalize some recent results obtained in [Y. Han and Q. Li, Threshold results for the existence of global and blow-up solutions to Kirchhoff equations with arbitrary initial energy, Computers and Mathematics with Applications, 75(9):3283-3297, 2018].
\end{abstract}

Keywords: $p$-Kirchhoff, potential well, global existence, blow up, initial energy.

AMS Subject Classification: 35K20; 35K92.

\section{Introduction}

In this paper, we study the global existence and finite time blow-up of solutions to the following parabolic type $p$-Kirchhoff initial boundary value problem

$$
\begin{cases}u_{t}-\left(a+b \int_{\Omega}|\nabla u|^{p} \mathrm{~d} x\right) \Delta_{p} u=|u|^{q-1} u, & (x, t) \in \Omega \times(0, T), \\ u=0, & (x, t) \in \partial \Omega \times(0, T), \\ u(x, 0)=u_{0}(x), & x \in \Omega .\end{cases}
$$

Copyright (C) 2019 The Author(s). Published by VGTU Press

This is an Open Access article distributed under the terms of the Creative Commons Attribution License (http://creativecommons.org/licenses/by/4.0/), which permits unrestricted use, distribution, and reproduction in any medium, provided the original author and source are credited. 
Here $a, b$ are two positive constants, $\Delta_{p} u=\operatorname{div}\left(|\nabla u|^{p-2} \nabla u\right)$ is the standard $p$-Laplace operator with $p>\max \{2 n /(n+2), 1\}, 2 p-1<q<p^{*}-1$, where $p^{*}$ is the Sobolev conjugate of $p$, i.e. $p^{*}=+\infty$ for $n \leq p$ and $p^{*}=n p /(n-p)$ for $n>p . \Omega \subset \mathbb{R}^{n}(n \geq 1)$ is a bounded smooth domain with the boundary $\partial \Omega$, Moreover, $u_{0} \in W_{0}^{1, p}(\Omega)$.

In the recent years, much effort has been devoted to nonlocal problems because of their wide applications in both physics and biology. For example, when the length changes of the string produced by transverse vibrations is taken into account, the classical D'Alembert wave equation for free vibrations of elastic strings is replaced by (see [8])

$$
\varepsilon u_{t t}^{\varepsilon}+u_{t}^{\varepsilon}-M\left(\int_{\Omega}\left|\nabla u^{\varepsilon}\right|^{p} d x\right) \Delta_{p} u^{\varepsilon}=f\left(x, t, u^{\varepsilon}\right),
$$

where $M(s)=a+b s, a, b>0$ and $p>1$. For $p=2$, such nonlocal equations were first proposed by Kirchhoff [11] in 1883 and therefore were usually referred to as Kirchhoff equations. The existence, uniqueness and regularities of solutions to Kirchhoff type equations were well studied since the pioneer work of Lions [15]. We refer the interested reader to, for example, $[4,5,17]$ and the references therein. By taking $\varepsilon=0$ formally, (1.2) becomes a Kirchhoff type parabolic equation

$$
u_{t}-M\left(\int_{\Omega}|\nabla u|^{p} d x\right) \Delta_{p} u=f(x, t, u) .
$$

Problem (1.3) can also be used to describe the motion of a nonstationary fluid or gas in a nonhomogeneous and anisotropic medium, and the nonlocal term $M$ appearing in (1.3) can describe a possible change in the global state of the fluid or gas caused by its motion in the considered medium [6].

When $f(x, t, u) \equiv f(x)$ and $0<m \leq M(s) \leq M_{0}$ for all $s \geq 0$, Chipot et al. investigated the existence, uniqueness and asymptotic behavior of solutions to (1.3) for both $p=2$ and general $p>1$ (see $[2,3]$ ). The stationary problem associated with (1.3) was also investigated in detail by using variational methods. On the other hand, when the nonlinearity $f$ depends on the unknown $u$ and grows super-linearly with respect to $u$ as it tends to infinity, the solutions to (1.3) might blow up in finite time. Recently, Han and Li [10] considered the global existence and finite time blow-up properties of solutions to (1.3) with $f(x, t, u)$ replaced by $|u|^{q-1} u$ when $p=2$ (under homogeneous Dirichlet boundary condition). By applying the potential well method first proposed by Sattinger et al. [19,22] and then improved by Liu and $\mathrm{Xu}[16,24]$, they obtained a threshold result for the solutions to (1.1) to exist globally or to blow up in finite time when the initial energy is smaller than or equal to the depth of the potential well. The decay rates of the global solutions were also derived. Moreover, some sufficient conditions for the existence of global and finite time blow-up solutions were also given for supercritical initial energy, by using some variational tricks.

Inspired by some ideas from $[6,10,13,21,24]$, we shall consider the global existence and finite time blow-up of solutions to problem (1.1) for general $p>1$, by combining the modified potential well method with the classical Galerkin's 
approximation and energy estimates. It is noteworthy that the results obtained here are not trivial generalization of that of the case $p=2$ in [10]. The main difficulty is of course brought by the diffusion term, a combination of a nonlocal term and a $p$-Laplacian, which usually prevent us from obtaining the convergence $\left\|\nabla u_{n}\right\|_{p} \rightarrow\|\nabla u\|_{p}$ by the boundedness of the approximation solutions $\left\{u_{n}\right\}$ in $W_{0}^{1, p}(\Omega)$. To overcome this difficulty, we will make full use of the monotonicity of the nonlocal operator to recover the strong convergence (see Theorem 1). In addition, by applying the concavity arguments introduced by Levine [12] together with the properties of potential wells, we obtain the existence of finite time blow-up solutions under proper initial conditions. When the initial energy is supercritical, we will give some sufficient conditions for problem (1.1) to admit solutions that vanish at infinity or solutions that blow up in finite time, in terms of two variational numbers. As a byproduct we show that for any $M>d$, there exists a $u_{0}$ such that $J\left(u_{0}\right)>M$ and that the solutions to problem (1.1) with $u_{0}$ as initial datum blow up in finite time.

It is worth pointing out that there are also some important works on Kirchhoff type problems involving fractional Laplacian or $p$-Laplacian, among which we only mention $[18,20,23]$, where local and global well-posedness, long time behaviors and finite time blow-up of weak solutions are investigated, under some appropriate conditions.

The rest of this paper is organized as follows. In Section 2, some notations, definitions, functionals and sets as well as some lemmas concerning their basic properties are presented. Sections 3 and 4 will be devoted to the cases $J\left(u_{0}\right)<$ $d$ and $J\left(u_{0}\right)=d$, respectively, and in Section 5 , we shall consider the case $J\left(u_{0}\right)>d$, where $J(u)$ is the potential energy functional that will be defined in Section 2.

\section{Preliminaries}

In this paper, we denote by $\|u\|_{r}$ the $L^{r}(\Omega)$ norm of a Lebesgue function $u \in L^{r}(\Omega)$ for $1 \leq r \leq \infty$, and by $(\cdot, \cdot)$ the inner product in $L^{2}(\Omega)$. We will equip $W_{0}^{1, p}(\Omega)$ with the norm $\|u\|_{W_{0}^{1, p}(\Omega)}=\|\nabla u\|_{p}$, which is equivalent to the standard one due to Poincaré's inequality. Before stating the main results, we first introduce some notations and definitions of some functionals and sets, and then investigate their basic properties. For $u \in W_{0}^{1, p}(\Omega)$, define

$$
\begin{aligned}
J(u) & =\frac{a}{p}\|\nabla u\|_{p}^{p}+\frac{b}{2 p}\|\nabla u\|_{p}^{2 p}-\frac{1}{q+1}\|u\|_{q+1}^{q+1},(\text { the potential energy functional }) \\
I(u) & =a\|\nabla u\|_{p}^{p}+b\|\nabla u\|_{p}^{2 p}-\|u\|_{q+1}^{q+1},(\text { the Nehari's functional) }
\end{aligned}
$$

and the Nehari's manifolds

$$
\begin{aligned}
& \mathcal{N}=\left\{u \in W_{0}^{1, p}(\Omega) \mid I(u)=0,\|\nabla u\|_{p} \neq 0\right\} \\
& \mathcal{N}_{+}=\left\{u \in W_{0}^{1, p}(\Omega) \mid I(u)>0\right\}, \quad \mathcal{N}_{-}=\left\{u \in W_{0}^{1, p}(\Omega) \mid I(u)<0\right\}
\end{aligned}
$$


Both $J(u)$ and $I(u)$ are well defined and continuous in $W_{0}^{1, p}(\Omega)$ since $q+1<p^{*}$. The potential well and its corresponding set are defined respectively by

$$
\begin{aligned}
W & =\left\{u \in W_{0}^{1, p}(\Omega) \mid I(u)>0, J(u)<d\right\} \cup\{0\}, \\
V & =\left\{u \in W_{0}^{1, p}(\Omega) \mid I(u)<0, J(u)<d\right\},
\end{aligned}
$$

where $d$ is the depth of the potential well that can be characterized by

$$
d=\inf _{0 \neq u \in W_{0}^{1, p}(\Omega)} \sup _{\lambda \geq 0} J(\lambda u)=\inf _{u \in \mathcal{N}} J(u) .
$$

The positivity of $d$ is given in Lemma 1 .

Lemma 1. The depth $d$ of the potential well $W$ is positive.

Proof. Since $q+1<p^{*}, W_{0}^{1, p}(\Omega)$ can be embedded into $L^{q+1}(\Omega)$ continuously. Denote by $S>0$ the best embedding constant, i.e. $\|u\|_{q+1} \leq S\|\nabla u\|_{p}, \quad \forall u \in$ $W_{0}^{1, p}(\Omega)$. Therefore, for any $u \in \mathcal{N}$,

$$
a\|\nabla u\|_{p}^{p}+b\|\nabla u\|_{p}^{2 p}=\|u\|_{q+1}^{q+1} \leq S^{q+1}\|\nabla u\|_{p}^{q+1}
$$

which implies $\|\nabla u\|_{p} \geq\left(\frac{a}{S^{q+1}}\right)^{1 /(q+1-p)}$. Recalling that $q+1>2 p$, we have

$$
\begin{aligned}
J(u) & =\frac{a}{p}\|\nabla u\|_{p}^{p}+\frac{b}{2 p}\|\nabla u\|_{p}^{2 p}-\frac{1}{q+1}\left(a\|\nabla u\|_{p}^{p}+b\|\nabla u\|_{p}^{2 p}\right) \\
& =\frac{a(q+1-p)}{p(q+1)}\|\nabla u\|_{p}^{p}+\frac{b(q+1-2 p)}{2 p(q+1)}\|\nabla u\|_{p}^{2 p} \\
& \geq \frac{a(q+1-p)}{p(q+1)}\left(\frac{a}{S^{q+1}}\right)^{p /(q+1-p)}+\frac{b(q+1-2 p)}{2 p(q+1)}\left(\frac{a}{S^{q+1}}\right)^{2 p /(q+1-p)} .
\end{aligned}
$$

Therefore,

$$
d \geq \frac{a(q+1-p)}{p(q+1)}\left(\frac{a}{S^{q+1}}\right)^{p /(q+1-p)}+\frac{b(q+1-2 p)}{2 p(q+1)}\left(\frac{a}{S^{q+1}}\right)^{2 p /(q+1-p)} .
$$

The proof is complete.

Next, for any $\delta>0$, define the modified Nehari's functional and Nehari's manifold as follows:

$$
\begin{aligned}
I_{\delta}(u) & =\delta\left(a+b\|\nabla u\|_{p}^{p}\right)\|\nabla u\|_{p}^{p}-\|u\|_{q+1}^{q+1}, \\
\mathcal{N}_{\delta} & =\left\{u \in W_{0}^{1, p}(\Omega) \mid I_{\delta}(u)=0,\|\nabla u\|_{p} \neq 0\right\} .
\end{aligned}
$$

The modified potential wells and their corresponding sets are defined respectively by

$$
\begin{aligned}
W_{\delta} & =\left\{u \in W_{0}^{1, p}(\Omega) \mid I_{\delta}(u)>0, J(u)<d(\delta)\right\} \cup\{0\} \\
V_{\delta} & =\left\{u \in W_{0}^{1, p}(\Omega) \mid I_{\delta}(u)<0, J(u)<d(\delta)\right\} .
\end{aligned}
$$


Here $d(\delta)=\inf _{u \in \mathcal{N}_{\delta}} J(u)$ is the potential depth of $W_{\delta}$, which is also positive.

For any $s>d$, define the (closed) sublevels of $J$ by

$$
J^{s}=\left\{u \in W_{0}^{1, p}(\Omega) \mid J(u) \leq s\right\} .
$$

By the definition of $J(u), \mathcal{N}, J^{s}$ and $d$, we see that

$$
\begin{aligned}
\mathcal{N}^{s} & \triangleq \mathcal{N} \cap J^{s}=\left\{u \in \mathcal{N}: \frac{a(q+1-p)}{p(q+1)}\|\nabla u\|_{p}^{p}+\frac{b(q+1-2 p)}{2 p(q+1)}\|\nabla u\|_{p}^{2 p} \leq s\right\} \\
& \neq \emptyset
\end{aligned}
$$

We also define two variational numbers

$$
\lambda_{s}=\inf \left\{\|u\|_{2} \mid u \in \mathcal{N}^{s}\right\}, \quad \Lambda_{s}=\sup \left\{\|u\|_{2} \mid u \in \mathcal{N}^{s}\right\}
$$

It is clear that $\lambda_{s}$ is nonincreasing in $s$ and $\Lambda_{s}$ is nondecreasing in $s$.

Finally we introduce the following sets

$$
\begin{gathered}
\mathcal{B}=\left\{u_{0} \in W_{0}^{1, p}(\Omega) \mid \text { the solution } u(t) \text { to }(1.1) \text { blows up in finite time }\right\}, \\
\mathcal{G}_{0}=\left\{u_{0} \in W_{0}^{1, p}(\Omega) \mid \text { the solution } u(t) \text { to }(1.1) \text { tends to } 0 \text { in } W_{0}^{1, p}(\Omega)\right. \\
\text { as } t \rightarrow \infty\} .
\end{gathered}
$$

When $u_{0} \in \mathcal{G}_{0}$, we say that the solutions to problem (1.1) vanish at infinity.

Since the equation in (1.1) is singular or degenerate when $p \neq 2$, classical solutions may not exist in general. Therefore, we give the definition of weak solutions.

Definition 1. (Weak solution) A function $u=u(x, t) \in L^{\infty}\left(0, T ; W_{0}^{1, p}(\Omega)\right)$ with $u_{t} \in L^{2}\left(0, T ; L^{2}(\Omega)\right)$ is called a weak solution of problem (1.1) on $\Omega \times$ $[0, T)$, if $u(x, 0)=u_{0} \in W_{0}^{1, p}(\Omega)$ and satisfies

$$
\left(u_{t}, \phi\right)+\left(\left(a+b \int_{\Omega}|\nabla u|^{p} \mathrm{~d} x\right)|\nabla u|^{p-2} \nabla u, \nabla \phi\right)=\left(|u|^{q-1} u, \phi\right), \text { a.e. } t \in(0, T),
$$

for any $\phi \in W_{0}^{1, p}(\Omega)$. Moreover, $u(x, t)$ satisfies

$$
\int_{0}^{t}\left\|u_{\tau}\right\|_{2}^{2} \mathrm{~d} \tau+J(u(x, t))=J\left(u_{0}\right), \quad \text { a. e. } t \in(0, T) .
$$

The following lemmas show some basic properties of the functionals and sets defined above, and will play a fundamental role in the proof of the main results. Most of the proofs are more or less standard and hence are omitted. Interested readers may refer to $[10,24]$ for the details.

Lemma 2. Let $2 p-1<q<p^{*}-1$. Then for any $u \in W_{0}^{1, p}(\Omega),\|\nabla u\|_{p} \neq 0$, we have

(i) $\lim _{\lambda \rightarrow 0^{+}} J(\lambda u)=0, \lim _{\lambda \rightarrow+\infty} J(\lambda u)=-\infty$.

(ii) there exists a unique $\lambda^{*}=\lambda^{*}(u)>0$ such that $\left.\frac{d}{d \lambda} J(\lambda u)\right|_{\lambda=\lambda^{*}}=0$. $J(\lambda u)$ is increasing on $0<\lambda \leq \lambda^{*}$, decreasing on $\lambda^{*} \leq \lambda<+\infty$ and takes its maximum at $\lambda=\lambda^{*}$. 
(iii) $I(\lambda u)>0$ on $0<\lambda<\lambda^{*}, I(\lambda u)<0$ on $\lambda^{*}<\lambda<+\infty$ and $I\left(\lambda^{*} u\right)=0$.

Lemma 3. Let $2 p-1<q<p^{*}-1, u \in W_{0}^{1, p}(\Omega)$ and $r(\delta)=\left(\frac{\delta b}{S^{q+1}}\right)^{1 /(q+1-2 p)}$ ( $S$ is the constant given in Lemma 1). Then we have

(i) If $0 \leq\|\nabla u\|_{p} \leq r(\delta)$, then $I_{\delta}(u) \geq 0$.

(ii) If $I_{\delta}(u)<0$, then $\|\nabla u\|_{p}>r(\delta)$.

(iii) If $I_{\delta}(u)=0$, then $\|\nabla u\|_{p}=0$ or $\|\nabla u\|_{p} \geq r(\delta)$.

Lemma 4. The function $d(\delta)$ satisfies the following properties:

(i) $\lim _{\delta \rightarrow 0^{+}} d(\delta)=0, \lim _{\delta \rightarrow+\infty} d(\delta)=-\infty$.

(ii) $d(\delta)$ is increasing on $0<\delta \leq 1$, decreasing on $\delta \geq 1$, and takes its maximum $d=d(1)$ at $\delta=1$.

Lemma 5. Assume $u \in W_{0}^{1, p}(\Omega), 0<J(u)<d$, and $\delta_{1}<1<\delta_{2}$ are the two roots of the equation $d(\delta)=J(u)$. Then the sign of $I_{\delta}(u)$ does not change for $\delta_{1}<\delta<\delta_{2}$.

Lemma 6. Assume that $u(x, t)$ is a weak solution to problem (1.1) with $0<$ $J\left(u_{0}\right)<d$ and $T$ is the maximal existence time. Let $\delta_{1}<1<\delta_{2}$ be the two roots of the equation $d(\delta)=J\left(u_{0}\right)$.

(i) If $I\left(u_{0}\right)>0$, then $u(x, t) \in W_{\delta}$ for $\delta_{1}<\delta<\delta_{2}$ and $0<t<T$.

(ii) If $I\left(u_{0}\right)<0$, then $u(x, t) \in V_{\delta}$ for $\delta_{1}<\delta<\delta_{2}$ and $0<t<T$.

Lemma 7. Let $2 p<q+1<p^{*}$. Then

(i) 0 is away from both $\mathcal{N}$ and $\mathcal{N}_{-}$, i.e. $\operatorname{dist}(0, \mathcal{N})>0$, $\operatorname{dist}\left(0, \mathcal{N}_{-}\right)>0$.

(ii) For any $s>0$, the set $J^{s} \cap \mathcal{N}_{+}$is bounded in $W_{0}^{1, p}(\Omega)$.

Proof. (i) For any $u \in \mathcal{N}$, by the definition of $d$ we have

$$
\begin{aligned}
d \leq J(u) & =\frac{a}{p}\|\nabla u\|_{p}^{p}+\frac{b}{2 p}\|\nabla u\|_{p}^{2 p}-\frac{1}{q+1}\left(a\|\nabla u\|_{p}^{p}+b\|\nabla u\|_{p}^{2 p}\right) \\
& =\frac{a(q+1-p)}{p(q+1)}\|\nabla u\|_{p}^{p}+\frac{b(q+1-2 p)}{2 p(q+1)}\|\nabla u\|_{p}^{2 p} .
\end{aligned}
$$

Noticing that $q+1>2 p$, the above inequality implies that there exists a constant $c_{0}>0$ such that $\operatorname{dist}(0, \mathcal{N})=\inf _{u \in \mathcal{N}}\|\nabla u\|_{p} \geq c_{0}$.

For any $u \in \mathcal{N}_{-}$, we have $\|\nabla u\|_{p} \neq 0$, which implies that

$$
a\|\nabla u\|_{p}^{p}<a\|\nabla u\|_{p}^{p}+b\|\nabla u\|_{p}^{2 p}<\|u\|_{q+1}^{q+1} \leq S^{q+1}\|\nabla u\|_{p}^{q+1},
$$

or equivalently

$$
\|\nabla u\|_{p} \geq\left(\frac{a}{S^{q+1}}\right)^{1 /(q+1-p)} .
$$

Here $S>0$ is given in Lemma 1. Therefore, $\operatorname{dist}\left(0, \mathcal{N}_{-}\right)=\inf _{u \in \mathcal{N}_{-}}\|\nabla u\|_{p}>0$. 
(ii) For any $u \in J^{s} \cap \mathcal{N}_{+}$, we have $J(u) \leq s$ and $I(u)>0$. Therefore,

$$
\begin{aligned}
s \geq J(u) & =\frac{a(q+1-p)}{p(q+1)}\|\nabla u\|_{p}^{p}+\frac{b(q+1-2 p)}{2 p(q+1)}\|\nabla u\|_{p}^{2 p}+\frac{1}{q+1} I(u) \\
& >\frac{a(q+1-p)}{p(q+1)}\|\nabla u\|_{p}^{p}
\end{aligned}
$$

which yields

$$
\|\nabla u\|_{p}^{p}<\frac{p(q+1) s}{a(q+1-p)} .
$$

The proof is complete.

Lemma 8. Let $2 p<q+1<p^{*}$. Then for any $s>d, \lambda_{s}$ and $\Lambda_{s}$ defined in (2.2) satisfy

$$
0<\lambda_{s} \leq \Lambda_{s}<+\infty
$$

Proof. The following Gagliardo-Nirenberg's inequality for $u \in W_{0}^{1, p}(\Omega)$ is needed when showing the positivity of $\lambda_{s}$ (see [1] page 241),

$$
\|u\|_{q+1}^{q+1} \leq C\|\nabla u\|_{p}^{\alpha(q+1)}\|u\|_{2}^{(1-\alpha)(q+1)}, \quad \forall u \in W_{0}^{1, p}(\Omega),
$$

where $\alpha$ is determined by $\left(\frac{1}{2}+\frac{1}{n}-\frac{1}{p}\right) \alpha=\frac{1}{2}-\frac{1}{q+1}$ and $C$ is a positive constant depending only on $n, p$ and $q$. Since $p>2 n /(n+2)$ and $2<2 p<q+1<p^{*}$, it is easy to check that $\alpha \in(0,1)$. Therefore, for any $s>d$ and $u \in \mathcal{N}^{s}$, it follows from (2.6) that

$$
a\|\nabla u\|_{p}^{p}<\|u\|_{q+1}^{q+1} \leq C\|\nabla u\|_{p}^{\alpha(q+1)}\|u\|_{2}^{(1-\alpha)(q+1)},
$$

which then guarantees that

$$
a\|\nabla u\|_{p}^{p-\alpha(q+1)} \leq C\|u\|_{2}^{(1-\alpha)(q+1)} .
$$

By Lemma 7 (i) and (2.1) we see that the left-hand side of (2.7) is bounded away from 0 no matter what the sign of $p-\alpha(q+1)$ is. This proves $\lambda_{s}>0$. The fact that $\Lambda_{s}<\infty$ just follows from (2.1) and the Sobolev embedding inequality $\|u\|_{2} \leq C_{*}\|\nabla u\|_{p}$ since $p>2 n /(n+2)$ is equivalent to $2<p^{*}$. The proof is complete.

In the last part of this section, we investigate some basic properties of the nonlocal $p$-Laplacian $-\left(a+b\|\nabla u\|_{p}^{p}\right) \Delta_{p} u$ in (1.1), which will be used to prove the uniqueness of bounded weak solutions and are also of independent interest.

Consider the following functional:

$$
E(u)=\left(\frac{a}{p}+\frac{b}{2 p} \int_{\Omega}|\nabla u|^{p} \mathrm{~d} x\right) \int_{\Omega}|\nabla u|^{p} \mathrm{~d} x, \quad u \in W_{0}^{1, p}(\Omega) .
$$

It is easy to see that $E \in C^{1}\left(W_{0}^{1, p}(\Omega), \mathbb{R}\right)$, and the nonlocal operator is the Fréchet derivative operator of $E$ in the weak sense. 
Denote $L=E^{\prime}: W_{0}^{1, p}(\Omega) \rightarrow W^{-1, q}(\Omega)$, then $\forall u, v \in W_{0}^{1, p}(\Omega)$ we have

$$
\begin{gathered}
\langle L(u), v\rangle=\left(a+b\|\nabla u\|_{p}^{p}\right) \int_{\Omega}|\nabla u|^{p-2} \nabla u \cdot \nabla v d x \\
=M\left(\|\nabla u\|_{p}^{p}\right) \int_{\Omega}|\nabla u|^{p-2} \nabla u \cdot \nabla v d x .
\end{gathered}
$$

Here $q$ is the Hölder's conjugate of $p$ and $\langle$,$\rangle denotes the pairing between$ $W^{-1, q}(\Omega)$ and $W_{0}^{1, p}(\Omega)$.

Lemma 9. The nonlocal p-Laplacian $L$ has the following properties:

(i) $L: W_{0}^{1, p}(\Omega) \rightarrow W^{-1, q}(\Omega)$ is a continuous, bounded and satisfies for any $u, v \in W_{0}^{1, p}(\Omega)$ that

$$
\begin{gathered}
\langle L(u)-L(v), u-v\rangle \geq\left[M\left(\|\nabla u\|_{p}^{p}\right)\|\nabla u\|_{p}^{p-1}\right. \\
\left.-M\left(\|\nabla v\|_{p}^{p}\right)\|\nabla v\|_{p}^{p-1}\right]\left(\|\nabla u\|_{p}-\|\nabla v\|_{p}\right) .
\end{gathered}
$$

(ii) $L$ is a mapping of type $S_{+}$, i.e. if $u_{n} \rightarrow u$ weakly in $W_{0}^{1, p}(\Omega)$ and $\overline{\lim }_{n \rightarrow \infty}\left\langle L\left(u_{n}\right), u_{n}-u\right\rangle \leq 0$, then $u_{n} \rightarrow u$ strongly in $W_{0}^{1, p}(\Omega)$.

Proof. (i) It is obvious that $L$ is continuous and bounded. For any $u, v \in$ $W_{0}^{1, p}(\Omega)$, by using Hölder's inequality we have

$$
\begin{aligned}
& \langle L(u)-L(v), u-v\rangle=\int_{\Omega}\left(M\left(\|\nabla u\|_{p}^{p}\right)|\nabla u|^{p-2} \nabla u-M\left(\|\nabla v\|_{p}^{p}\right)|\nabla v|^{p-2} \nabla v\right) \\
& \times(\nabla u-\nabla v) d x=M\left(\|\nabla u\|_{p}^{p}\right)\|\nabla u\|_{p}^{p}-M\left(\|\nabla u\|_{p}^{p}\right) \int_{\Omega}|\nabla u|^{p-2} \nabla u \nabla v d x \\
& \quad+M\left(\|\nabla v\|_{p}^{p}\right)\|\nabla v\|_{p}^{p}-M\left(\|\nabla v\|_{p}^{p}\right) \int_{\Omega}|\nabla v|^{p-2} \nabla v \nabla u d x \\
& \geq M\left(\|\nabla u\|_{p}^{p}\right)\|\nabla u\|_{p}^{p}-M\left(\|\nabla u\|_{p}^{p}\right)\|\nabla u\|_{p}^{p-1}\|\nabla v\|_{p} \\
& \quad+M\left(\|\nabla v\|_{p}^{p}\right)\|\nabla v\|_{p}^{p}-M\left(\|\nabla v\|_{p}^{p}\right)\|\nabla v\|_{p}^{p-1}\|\nabla u\|_{p} \\
& \geq\left[M\left(\|\nabla u\|_{p}^{p}\right)\|\nabla u\|_{p}^{p-1}-M\left(\|\nabla v\|_{p}^{p}\right)\|\nabla v\|_{p}^{p-1}\right]\left(\|\nabla u\|_{p}-\|\nabla v\|_{p}\right) .
\end{aligned}
$$

Therefore, $(2.8)$ is true. have

(ii) If $u_{n} \rightarrow u$ weakly in $W_{0}^{1, p}(\Omega)$ and $\varlimsup_{n \rightarrow \infty}\left\langle L\left(u_{n}\right), u_{n}-u\right\rangle \leq 0$, then we

$$
\varlimsup_{n \rightarrow \infty}\left\langle L\left(u_{n}\right)-L(u), u_{n}-u\right\rangle \leq 0 .
$$

Noticing that $M(s)$ is strictly monotone with respect to $s$, we obtain from (2.8) and (2.9) that $\left\|\nabla u_{n}\right\|_{p} \rightarrow\|\nabla u\|_{p}$ as $n \rightarrow \infty$, which, together with the weak convergence $u_{n} \rightarrow u$ in $W_{0}^{1, p}(\Omega)$, implies that $u_{n} \rightarrow u$ strongly in $W_{0}^{1, p}(\Omega)$. Hence $L$ is an $S_{+}$operator. The proof is complete.

\section{$3 \quad \mathbf{J}\left(\mathbf{u}_{0}\right)<\mathbf{d}$}

In this section we consider the behaviors of the solution to problem (1.1) under the condition $J\left(u_{0}\right)<d$ and give the threshold result for the solutions to exist globally or to blow up in finite time. 
Theorem 1. (Global existence for $J\left(u_{0}\right)<$ d.) Assume that $a, b>0, p>$ $\max \left\{\frac{2 n}{n+2}, 1\right\}, 2 p-1<q<p^{*}-1$ and $u_{0} \in W_{0}^{1, p}(\Omega)$. If $J\left(u_{0}\right)<d$ and $I\left(u_{0}\right)>0$, then problem $(1.1)$ admits a global solution $u \in L^{\infty}\left(0, \infty ; W_{0}^{1, p}(\Omega)\right)$ with $u_{t} \in L^{2}\left(0, \infty ; L^{2}(\Omega)\right)$ and $u(t) \in W$ for $0 \leq t<\infty$. Moreover, $\|u\|_{2}^{2} \leq$ $\left[\left\|u_{0}\right\|_{2}^{2-2 p}+C^{*}(p-1) t\right]^{-1 /(p-1)}$, where $C^{*}>0$ will be given in the proof. In addition, the weak solution is unique when it is bounded.

Proof. We will divide the proof into three steps for the convenience of the readers.

Step 1. Global existence. Global existence of weak solutions will be proved by combining Galerkin's approximation with a priori estimates. Let $\left\{\phi_{j}(x)\right\}$ be a system of basis of $W_{0}^{1, p}(\Omega)$ which is orthogonal in $L^{2}(\Omega)$ and construct the approximate solutions $u^{m}(x, t)$ to problem (1.1)

$$
u^{m}(x, t)=\sum_{j=1}^{m} a_{j}^{m}(t) \phi_{j}(x), \quad m=1,2, \cdots
$$

satisfying for $j=1,2, \cdots, m$

$$
\begin{aligned}
& \left(u_{t}^{m}, \phi_{j}\right)+M\left(\left\|\nabla u^{m}\right\|_{p}^{p}\right)\left(\left|\nabla u^{m}\right|^{p-2} \nabla u^{m}, \nabla \phi_{j}\right)=\left(\left|u^{m}\right|^{q-1} u^{m}, \phi_{j}\right), \\
& u^{m}(x, 0)=\sum_{j=1}^{m} b_{j}^{m} \phi_{j}(x) \rightarrow u_{0}(x) \text { in } W_{0}^{1, p}(\Omega) .
\end{aligned}
$$

Multiplying (3.1) by $\frac{d}{d t} a_{j}^{m}(t)$, summing for $j$ from 1 to $m$, and integrating with respect to $t$ from 0 to $t$, we obtain

$$
\int_{0}^{t}\left\|u_{\tau}^{m}\right\|_{2}^{2} \mathrm{~d} \tau+J\left(u^{m}\right)=J\left(u^{m}(x, 0)\right), \quad 0 \leq t<\infty .
$$

Recalling the convergence of $u^{m}(x, 0) \rightarrow u_{0}(x)$ in $W_{0}^{1, p}(\Omega)$, we have

$$
J\left(u^{m}(x, 0)\right) \rightarrow J\left(u_{0}(x)\right)<d \quad \text { and } \quad I\left(u^{m}(x, 0)\right) \rightarrow I\left(u_{0}(x)\right)>0 .
$$

Thus, for sufficiently large $m$ and for any $0 \leq t<\infty$, we obtain

$$
\int_{0}^{t}\left\|u_{\tau}^{m}\right\|_{2}^{2} d \tau+J\left(u^{m}(x, t)\right)=J\left(u^{m}(x, 0)\right)<d \quad \text { and } \quad I\left(u^{m}(x, 0)\right)>0 .
$$

We first claim that for sufficiently large $m$ that $u^{m}(x, t) \in W$ for $0 \leq t<\infty$. Otherwise, there exists a $t_{0} \in(0, T)$ such that $u^{m}\left(x, t_{0}\right) \in \partial W$. Noticing that 0 is an interior point of $W$, we thus have

(i) $I\left(u^{m}\left(x, t_{0}\right)\right)=0,\left\|\nabla u^{m}\left(x, t_{0}\right)\right\|_{p} \neq 0$, or $(i i) \quad J\left(u^{m}\left(x, t_{0}\right)\right)=d$.

From (3.3) it follows that $J\left(u^{m}\left(x, t_{0}\right)\right)<d$. Therefore, case (ii) is impossible. On the other hand, if $I\left(u^{m}\left(x, t_{0}\right)\right)=0$ and $\left\|\nabla u^{m}\left(x, t_{0}\right)\right\|_{p} \neq 0$, then by the definition of $d$ we obtain $J\left(u^{m}\left(x, t_{0}\right)\right) \geq d$, again contradictive with (3.3). 
Since $u^{m}(x, t) \in W$ for sufficiently large $m$ and $0 \leq t<\infty$, we have $I\left(u^{m}(x, t)\right)>0$ for all $t \geq 0$. Then it follows from the following equality

$$
J\left(u^{m}\right)=\frac{a(q+1-p)}{p(q+1)}\left\|\nabla u^{m}\right\|_{p}^{p}+\frac{b(q+1-2 p)}{2 p(q+1)}\left\|\nabla u^{m}\right\|_{p}^{2 p}+\frac{1}{q+1} I\left(u^{m}\right)
$$

and (3.3) that

$$
\int_{0}^{t}\left\|u_{\tau}^{m}\right\|_{2}^{2} \mathrm{~d} \tau+\frac{a(q+1-p)}{p(q+1)}\left\|\nabla u^{m}\right\|_{p}^{p}+\frac{b(q+1-2 p)}{2 p(q+1)}\left\|\nabla u^{m}\right\|_{p}^{2 p}<d,
$$

for sufficiently large $m$ and for any $0 \leq t<\infty$, which then implies

$$
\begin{aligned}
& \left\|u^{m}\right\|_{W_{0}^{1, p}(\Omega)}^{p} \leq \frac{d p(q+1)}{a(q+1-p)}, \quad 0 \leq t<\infty, \\
& \int_{0}^{t}\left\|u_{\tau}^{m}\right\|_{2}^{2} d \tau<d, \quad 0 \leq t<\infty, \\
& \left\|\left|u^{m}\right|^{q-1} u^{m}\right\|_{(q+1) / q}=\left\|u^{m}\right\|_{q+1}^{q} \leq S^{q}\left\|u^{m}\right\|_{W_{0}^{1, p}(\Omega)}^{q} \\
& \quad \leq S^{q}\left(\frac{d p(q+1)}{a(q+1-p)}\right)^{q / p}, \quad 0 \leq t<\infty .
\end{aligned}
$$

By (3.4) we also see that there exists a constant $C>0$, independent of $m$ and $t$ such that

$$
\begin{aligned}
& \left\|M\left(\left\|\nabla u^{m}\right\|_{p}^{p}\right)\left|\nabla u^{m}\right|^{p-2} \nabla u^{m}\right\|_{p /(p-1)} \\
& \quad=\left\|\left(a+b\left\|\nabla u^{m}\right\|_{p}^{p}\right)\left|\nabla u^{m}\right|^{p-2} \nabla u^{m}\right\|_{p /(p-1)} \leq C .
\end{aligned}
$$

Therefore, by (3.4)-(3.7), the standard diagonal method and Aubin-Lions' compactness embedding theorem [14]( since $p>2 n /(n+2))$, we get a subsequence of $\left\{u^{m}\right\}$ (still denoted by $\left\{u^{m}\right\}$ ) such that for each $T>0$, as $m \rightarrow \infty$,

$$
\left\{\begin{array}{l}
u_{t}^{m} \rightarrow u_{t}, \text { weakly in } L^{2}\left(0, T ; L^{2}(\Omega)\right), \\
u^{m} \rightarrow u, \text { weakly }{ }^{*} \text { in } L^{\infty}\left(0, T ; W_{0}^{1, p}(\Omega)\right), \\
u^{m} \rightarrow u, \text { strongly in } L^{2}(\Omega \times(0, T)) \text { and a.e. in } \Omega \times(0, T), \\
\left|u^{m}\right|^{q-1} u^{m} \rightarrow|u|^{q-1} u, \text { weakly in } L^{(q+1) / q}(\Omega \times(0, T)), \\
M\left(\left\|\nabla u^{m}\right\|_{p}^{p}\right)\left|\nabla u^{m}\right|^{p-2} \nabla u^{m} \rightarrow \xi, \text { weakly* in } L^{\infty}\left(0, T ; L^{\frac{p}{(p-1)}}(\Omega)\right) .
\end{array}\right.
$$

We will show that $u$ is a weak solution to problem (1.1) for any $T>0$. For this, fix $T>0$ and denote $Q_{T}=\Omega \times(0, T)$. First, since $p>2 n /(n+2)$, we see from (3.4) that $\int_{\Omega}\left|u^{m}(x, T)\right|^{2} \mathrm{~d} x \leq C$. Therefore, there exists a subsequence of $\left\{u^{m}(x, T)\right\}$ (which we still denote by $\left\{u^{m}(x, T)\right\}$ ) and a function $v \in L^{2}(\Omega)$ such that $u^{m}(x, T) \rightarrow v$ weakly in $L^{2}(\Omega)$. Then for any $\varphi(x) \in C_{0}^{\infty}(\Omega)$ and $\eta(t) \in C^{1}[0, T]$, it holds that

$$
\iint_{Q_{T}} u_{t}^{m} \varphi \eta \mathrm{d} x \mathrm{~d} t=\int_{\Omega}\left[u^{m}(x, T) \eta(T)-u^{m}(x, 0) \eta(0)\right] \varphi \mathrm{d} x-\iint_{Q_{T}} u^{m} \varphi \eta_{t} \mathrm{~d} x \mathrm{~d} t .
$$


By letting $m \rightarrow \infty$ in the above inequality, we obtain

$$
\int_{\Omega}(v-u(x, T)) \eta(T) \varphi \mathrm{d} x-\int_{\Omega}\left(u_{0}(x)-u(x, 0)\right) \eta(0) \varphi \mathrm{d} x=0 .
$$

Setting $\eta(T)=1, \eta(0)=0$ or $\eta(T)=0, \eta(0)=1$, and by the density of $C_{0}^{\infty}(\Omega)$ in $L^{2}(\Omega)$, we have $v=u(x, T)$ and $u(x, 0)=u_{0}(x)$ for almost every $x \in \Omega$. By the weakly lower semi-continuity of the $L^{2}(\Omega)$ norm we get

$$
\int_{\Omega} u^{2}(x, T) d x \leq \liminf _{m \rightarrow \infty} \int_{\Omega}\left|u^{m}(x, T)\right|^{2} d x .
$$

Next we will show that

$$
\lim _{m \rightarrow \infty} \iint_{Q_{T}}\left|u^{m}\right|^{q+1} d x d t=\iint_{Q_{T}}|u|^{q+1} d x d t .
$$

Recalling (3.4) and the fact that $q+1<p^{*}$, we have for any measurable subset $E \subset Q_{T}$ that

$$
\begin{aligned}
& \iint_{E}\left|u^{m}\right|^{q+1} d x d t \leq\left(\iint_{E}\left|u^{m}\right|^{p^{*}} d x d t\right)^{(q+1) / p^{*}}|E|^{\left(p^{*}-q-1\right) / p^{*}} \\
& \quad \leq\left(\iint_{Q_{T}}\left|u^{m}\right|^{p^{*}} d x d t\right)^{(q+1) / p^{*}}|E|^{\left(p^{*}-q-1\right) / p^{*}} \\
& \leq S_{1}^{q+1}\left[\int_{0}^{T}\left(\int_{\Omega}\left|\nabla u^{m}\right|^{p} d x\right)^{p^{*} / p} d t\right]^{(q+1) / p^{*}}|E|^{\left(p^{*}-q-1\right) / p^{*}} \leq C|E|^{\left(p^{*}-q-1\right) / p^{*}},
\end{aligned}
$$

which shows that $\left\{\left|u^{m}\right|^{q+1}\right\}_{m=1}^{\infty}$ is equi-integrable in $L^{1}\left(Q_{T}\right)$. Here $S_{1}>0$ is the embedding constant from $W_{0}^{1, p}(\Omega)$ to $L^{p^{*}}(\Omega)$ and $|E|$ is the Lebesgue's measure of $E$. Since $u^{m}(x, t) \rightarrow u(x, t)$ a.e. in $Q_{T}$, the Vitali's Theorem implies that (3.10) is true.

Set $V_{k}=\operatorname{span}\left\{\phi_{1}, \phi_{2}, \cdots, \phi_{k}\right\}$. Then for any $w \in C\left(0, T ; V_{k}\right)(k \leq m)$, it follows from (3.1) that

$$
\iint_{Q_{T}}\left[u_{t}^{m} w+M\left(\left\|\nabla u^{m}\right\|_{p}^{p}\right)\left|\nabla u^{m}\right|^{p-2} \nabla u^{m} \nabla w\right] d x d t=\iint_{Q_{T}}\left|u^{m}\right|^{q-1} u^{m} w d x d t .
$$

In particular, we have

$$
\iint_{Q_{T}}\left[u_{t}^{m} u^{m}+M\left(\left\|\nabla u^{m}\right\|_{p}^{p}\right)\left|\nabla u^{m}\right|^{p}\right] \mathrm{d} x \mathrm{~d} t=\iint_{Q_{T}}\left|u^{m}\right|^{q+1} \mathrm{~d} x \mathrm{~d} t .
$$

Letting $m \rightarrow \infty$ in (3.11) and noticing (3.8) we obtain

$$
\iint_{Q_{T}}\left[u_{t} w+\xi \nabla w\right] \mathrm{d} x \mathrm{~d} t=\iint_{Q_{T}}|u|^{q-1} u w \mathrm{~d} x \mathrm{~d} t .
$$

Choosing $w=u^{m}$ in (3.13), letting $m \rightarrow \infty$ and making use of (3.8) again we arrive at

$$
\frac{1}{2} \int_{\Omega}\left(u^{2}(x, T)-u_{0}^{2}(x)\right) \mathrm{d} x+\iint_{Q_{T}} \xi \nabla u \mathrm{~d} x \mathrm{~d} t=\iint_{Q_{T}}|u|^{q+1} \mathrm{~d} x \mathrm{~d} t .
$$


Our next goal is to show that $\xi=M\left(\|\nabla u\|_{p}^{p}\right)|\nabla u|^{p-2} \nabla u$. For this, set

$$
T_{m}=\iint_{Q_{T}} M\left(\left\|\nabla u^{m}\right\|_{p}^{p}\right)\left(\left|\nabla u^{m}\right|^{p-2} \nabla u^{m}-|\nabla u|^{p-2} \nabla u\right)\left(\nabla u^{m}-\nabla u\right) d x d t .
$$

Then $T_{m} \geq 0$. We aim to show that $T_{m} \rightarrow 0$ as $m \rightarrow \infty$, which in turn implies that $\nabla u^{m} \rightarrow \nabla u$ strongly in $\left(L^{p}\left(Q_{T}\right)\right)^{n}$. Indeed, it is easily seen by the definition of $T_{m}$ that

$$
a \iint_{Q_{T}}\left(\left|\nabla u^{m}\right|^{p-2} \nabla u^{m}-|\nabla u|^{p-2} \nabla u\right)\left(\nabla u^{m}-\nabla u\right) \mathrm{d} x \mathrm{~d} t \leq T_{m} .
$$

If $p \geq 2$, then

$$
\iint_{Q_{T}}\left(\left|\nabla u^{m}-\nabla u\right|^{p} d x d t \leq C \iint_{Q_{T}}\left(\left|\nabla u^{m}\right|^{p-2} \nabla u^{m}-|\nabla u|^{p-2} \nabla u\right)\left(\nabla u^{m}-\nabla u\right) d x d t .\right.
$$

If $2 n /(n+2)<p<2$, then

$$
\begin{aligned}
& \iint_{Q_{T}}\left(\left|\nabla u^{m}-\nabla u\right|^{p} d x d t \leq C \|\left[\left(\left|\nabla u^{m}\right|^{p-2} \nabla u^{m}-|\nabla u|^{p-2} \nabla u\right)\right.\right. \\
& \left.\quad \times\left(\nabla u^{m}-\nabla u\right)\right]^{p / 2}\left\|_{L^{2 / p}\left(Q_{T}\right)}\right\|\left(\left|\nabla u^{m}\right|^{p}+|\nabla u|^{p}\right)^{(2-p) / 2} \|_{L^{2 /(2-p)}\left(Q_{T}\right)} .
\end{aligned}
$$

Here the positive constant $C$ depends only on $p$. Noticing that $\|\left(\left|\nabla u^{m}\right|^{p}+\right.$ $\left.|\nabla u|^{p}\right)^{(2-p) / 2} \|_{L^{2 /(2-p)}\left(Q_{T}\right)}$ is uniformly bounded in $m$, we obtain the strong convergence $\nabla u^{m} \rightarrow \nabla u$ in $\left(L^{p}\left(Q_{T}\right)\right)^{n}$ from $T_{m} \rightarrow 0$ for both $p \geq 2$ and $2 n /(n+2)<p<2$.

By (3.12) we can rewrite $T_{m}$ as follows

$$
\begin{aligned}
T_{m}= & \iint_{Q_{T}}\left|u^{m}\right|^{q+1} \mathrm{~d} x \mathrm{~d} t-\frac{1}{2} \int_{\Omega}\left(\left|u^{m}(x, T)\right|^{2}-\left|u^{m}(x, 0)\right|^{2}\right) \mathrm{d} x \\
& -\iint_{Q_{T}} M\left(\left\|\nabla u^{m}\right\|_{p}^{p}\right)\left|\nabla u^{m}\right|^{p-2} \nabla u^{m} \nabla u \mathrm{~d} x \mathrm{~d} t \\
& -\iint_{Q_{T}} M\left(\left\|\nabla u^{m}\right\|_{p}^{p}\right)|\nabla u|^{p-2} \nabla u\left(\nabla u^{m}-\nabla u\right) \mathrm{d} x \mathrm{~d} t .
\end{aligned}
$$

It can be concluded from (3.4) that $\left\{M\left(\left\|\nabla u^{m}\right\|_{p}^{p}\right)\right\}_{m=1}^{\infty}$ in equi-integrable and uniformly bounded in $L^{1}(0, T)$. Thus, there exists a subsequence of $\left\{u^{m}\right\}$ (still denoted by $\left.\left\{u^{m}\right\}\right)$ and a measurable function $m(t)$ such that $M\left(\left\|\nabla u^{m}\right\|_{p}^{p}\right) \rightarrow$ $m(t)$ for almost a.e. $t \in(0, T)$. Since

$$
\left.\left.\left|M\left(\left\|\nabla u^{m}\right\|_{p}^{p}\right)\right| \nabla u\right|^{p-2} \nabla u\right|^{p /(p-1)} \leq C|\nabla u|^{p} \in L^{1}\left(Q_{T}\right),
$$

it can be deduced by Lebesgue's dominated convergence theorem that

$$
M\left(\left\|\nabla u^{m}\right\|_{p}^{p}\right)|\nabla u|^{p-2} \nabla u \rightarrow m(t)|\nabla u|^{p-2} \nabla u \text { strongly in }\left(L^{p /(p-1)}\left(Q_{T}\right)\right)^{n} \text {. }
$$


Therefore, by (3.9), (3.10), (3.14) and (3.15) we have

$$
\begin{aligned}
0 & \leq \limsup _{m \rightarrow \infty} T_{m} \\
& \leq \iint_{Q_{T}}\left(|u|^{q+1}-\xi \nabla u\right) \mathrm{d} x \mathrm{~d} t+\frac{1}{2} \int_{\Omega} u_{0}^{2}(x) \mathrm{d} x-\frac{1}{2} \int_{\Omega} u^{2}(x, T) \mathrm{d} x=0,
\end{aligned}
$$

which implies that $\lim _{m \rightarrow \infty} T_{m}=0$.

To complete the proof of Step 1, it remains to show $m(t)=M\left(\|\nabla u\|_{p}^{p}\right)$ and $\xi=M\left(\|\nabla u\|_{p}^{p}\right)|\nabla u|^{p-2} \nabla u$. The strong convergence of $\nabla u^{m} \rightarrow \nabla u$ in $\left(L^{p /(p-1)}\left(Q_{T}\right)\right)^{n}$ implies

$$
\begin{aligned}
& \int_{\Omega}\left|\nabla u^{m}-\nabla u\right|^{p} d x \rightarrow 0, \quad \int_{\Omega}\left|\nabla u^{m}\right|^{p} d x \rightarrow \int_{\Omega}|\nabla u|^{p} \mathrm{~d} x \text { a.e. in }(0, T), \\
& \nabla u^{m} \rightarrow \nabla u \text { a.e. in } Q_{T} .
\end{aligned}
$$

The fact that $m(t)=M\left(\|\nabla u\|_{p}^{p}\right)$ follows from (3.16). By (3.17) we have

$$
M\left(\left\|\nabla u^{m}\right\|_{p}^{p}\right)\left|\nabla u^{m}\right|^{p-2} \nabla u^{m} \rightarrow M\left(\|\nabla u\|_{p}^{p}\right)|\nabla u|^{p-2} \nabla u \quad \text { a.e. in } Q_{T},
$$

which, together with (3.8) implies that $\xi=M\left(\|\nabla u\|_{p}^{p}\right)|\nabla u|^{p-2} \nabla u$.

By (3.13) we obtain, for all $w \in C^{1}\left(0, T ; C_{0}^{\infty}(\Omega)\right)$ that

$$
\iint_{Q_{T}}\left[u_{t} w+M\left(\|\nabla u\|_{p}^{p}\right)|\nabla u|^{p-2} \nabla u \nabla w\right] \mathrm{d} x \mathrm{~d} t=\iint_{Q_{T}}|u|^{q-1} u w \mathrm{~d} x \mathrm{~d} t .
$$

By the arbitrariness of $w \in C^{1}\left(0, T ; C_{0}^{\infty}(\Omega)\right)$ and the density of $C_{0}^{\infty}(\Omega)$ in $W_{0}^{1, p}(\Omega)$, we see that

$$
\left(u_{t}, \phi\right)+\left(\left(a+b \int_{\Omega}|\nabla u|^{p} \mathrm{~d} x\right)|\nabla u|^{p-2} \nabla u, \nabla \phi\right)=\left(|u|^{q-1} u, \phi\right), \quad \text { a.e. } t \in(0, T),
$$

for any $\phi \in W_{0}^{1, p}(\Omega)$.

To prove (2.4) we first assume that $u(x, t)$ is smooth enough such that $u_{t} \in L^{2}\left(0, T ; W_{0}^{1, p}(\Omega)\right)$. Choosing $\phi=u_{t}$ as a test function and integrating (2.3) over $[0, t]$ one sees that $(2.4)$ is true. By the density of $L^{2}\left(0, T ; W_{0}^{1, p}(\Omega)\right)$ in $L^{2}(\Omega \times(0, T))$ it is known that (2.4) also holds for weak solutions of (1.1). Therefore $u$ is a global weak solution of problem (1.1).

Step 2. Decay rate. Taking $\phi=u$ in (2.1), we get

$$
\frac{1}{2} \frac{d}{d t}\|u\|_{2}^{2}=\left(u_{t}, u\right)=-a\|\nabla u\|_{p}^{p}-b\|\nabla u\|_{p}^{2 p}+\|u\|_{q+1}^{q+1}=-I(u) .
$$

From Lemma 6 it follows that $u(x, t) \in W_{\delta}$ for $\delta_{1}<\delta<\delta_{2}$ and $0<t<\infty$ under the condition $J\left(u_{0}\right)<d$ and $I\left(u_{0}\right)>0$. Thus we have $I_{\delta_{1}}(u) \geq 0$ for $0<t<\infty$. Therefore,

$$
\begin{aligned}
\frac{1}{2} \frac{d}{d t}\|u\|_{2}^{2} & =-I(u)=a\left(\delta_{1}-1\right)\|\nabla u\|_{p}^{p}+b\left(\delta_{1}-1\right)\|\nabla u\|_{p}^{2 p}-I_{\delta_{1}}(u) \\
& \leq \frac{b\left(\delta_{1}-1\right)}{S_{2}^{2 p}}\|u\|_{2}^{2 p}
\end{aligned}
$$


where $S_{2}>0$ is the best embedding constant from $W_{0}^{1, p}(\Omega)$ to $L^{2}(\Omega)$. Integrating the above inequality over $[0, t]$ we see that

$$
\|u\|_{2}^{2} \leq\left[\left\|u_{0}\right\|_{2}^{2-2 p}+C^{*}(p-1) t\right]^{-1 /(p-1)}
$$

where $C^{*}=2 b\left(1-\delta_{1}\right) / S_{2}^{2 p}>0$.

Step 3. Uniqueness of bounded solution. To prove the uniqueness of bounded weak solution, we assume that both $u$ and $v$ are bounded weak solutions of problem (1.1). Then, for any $\varphi \in W_{0}^{1, p}(\Omega)$, we have

$$
\begin{aligned}
& \left(u_{t}, \varphi\right)+M\left(\|\nabla u\|_{p}^{p}\right)\left(|\nabla u|^{p-2} \nabla u, \nabla \varphi\right)=\left(|u|^{q-1} u, \varphi\right), \\
& \left(v_{t}, \varphi\right)+M\left(\|\nabla v\|_{p}^{p}\right)\left(|\nabla v|^{p-2} \nabla v, \nabla \varphi\right)=\left(|v|^{q-1} v, \varphi\right) .
\end{aligned}
$$

Subtracting the above two equalities, taking $\varphi=u-v \in W_{0}^{1, p}(\Omega)$, integrating over $(0, t)$ for any $t>0$ and recalling $(2.8)$, we obtain

$$
\begin{aligned}
& \iint_{Q_{t}}(u-v)_{\tau}^{2} d x d \tau+\int_{0}^{t}\left[M\left(\|\nabla u\|_{p}^{p}\right)\|\nabla u\|_{p}^{p-1}-M\left(\|\nabla v\|_{p}^{p}\right)\|\nabla v\|_{p}^{p-1}\right] \\
& \quad \times\left(\|\nabla u\|_{p}-\|\nabla v\|_{p}\right) d \tau \leq \iint_{Q_{t}}\left(|u|^{q-1} u-|v|^{q-1} v\right)(u-v) d x d \tau .
\end{aligned}
$$

Since $(u-v)(x, 0)=0$ and the second part of the left hand side of (3.18) is nonnegative, we obtain, with the help of the boundedness of $u$ and $v$, that

$$
\int_{\Omega}(u-v)^{2}(x, t) \mathrm{d} x \leq C \iint_{Q_{t}}(u-v)^{2}(x, t) \mathrm{d} x \mathrm{~d} t,
$$

where $C>0$ is a constant depending only on $q$ and the bound of $u, v$. It then follows from Gronwall's inequality that

$$
\int_{\Omega}(u-v)^{2}(x, t) d x=0 .
$$

Thus $u=v$ a.e. in $\Omega \times(0, \infty)$ and the whole proof is complete.

Theorem 2. (Blow-up for $J\left(u_{0}\right)<$ d.) Assume $a, b>0, p>\max \{2 n /(n+$ $2), 1\}, 2 p-1<q<p^{*}-1$ and $u_{0} \in W_{0}^{1, p}(\Omega)$. Let $u$ be a weak solution of problem (1.1) with $u_{0} \in W_{0}^{1, p}(\Omega)$. If $J\left(u_{0}\right)<d$ and $I\left(u_{0}\right)<0$, then there exists a finite time $T$ such that $u$ blows up at $T$ in the sense that

$$
\lim _{t \rightarrow T} \int_{0}^{t}\|u\|_{2}^{2} d \tau=+\infty
$$

Proof. Suppose that $u$ is a global weak solution of problem (1.1) with $J\left(u_{0}\right)<$ $d, I\left(u_{0}\right)<0$ and define

$$
M(t)=\int_{0}^{t}\|u\|_{2}^{2} d \tau
$$


then

$$
\begin{aligned}
& M^{\prime}(t)=\|u\|_{2}^{2}, \\
& M^{\prime \prime}(t)=2\left(u_{t}, u\right)=-2\left(a\|\nabla u\|_{p}^{p}+b\|\nabla u\|_{p}^{2 p}-\|u\|_{q+1}^{q+1}\right)=-2 I(u) .
\end{aligned}
$$

Notice that

$$
J(u)=\frac{a(q+1-p)}{p(q+1)}\|\nabla u\|_{p}^{p}+\frac{b(q+1-2 p)}{2 p(q+1)}\|\nabla u\|_{p}^{2 p}+\frac{1}{q+1} I(u) .
$$

By (2.2), (3.20) and (3.21), we can get

$$
\begin{aligned}
M^{\prime \prime}(t)= & \frac{2 a(q+1-p)}{p}\|\nabla u\|_{p}^{p}+\frac{b(q+1-2 p)}{p}\|\nabla u\|_{p}^{2 p}-2(q+1) J(u) \\
= & \frac{2 a(q+1-p)}{p}\|\nabla u\|_{p}^{p}+\frac{b(q+1-2 p)}{p}\|\nabla u\|_{p}^{2 p} \\
& +2(q+1) \int_{0}^{t}\left\|u_{\tau}\right\|_{2}^{2} \mathrm{~d} \tau-2(q+1) J\left(u_{0}\right) \\
\geq & \frac{b(q+1-2 p)}{p}\|\nabla u\|_{p}^{2 p}+2(q+1) \int_{0}^{t}\left\|u_{\tau}\right\|_{2}^{2} \mathrm{~d} \tau-2(q+1) J\left(u_{0}\right) .
\end{aligned}
$$

Applying the basic inequality $s \leq s^{\alpha}+1$ for any $s>0$ and $\alpha>1$, we further have

$$
\begin{aligned}
\frac{M^{\prime \prime}(t)}{2(q+1)} & \geq \frac{b(q+1-2 p)}{2(q+1) p}\left(\|\nabla u\|_{p}^{2}-1\right)+\int_{0}^{t}\left\|u_{\tau}\right\|_{2}^{2} \mathrm{~d} \tau-J\left(u_{0}\right) \\
& \geq \frac{b(q+1-2 p)}{2(q+1) p S_{2}^{2}}\|u\|_{2}^{2}+\int_{0}^{t}\left\|u_{\tau}\right\|_{2}^{2} \mathrm{~d} \tau-\left[J\left(u_{0}\right)+\frac{b(q+1-2 p)}{2(q+1) p}\right] \\
& =\frac{b(q+1-2 p)}{2(q+1) p S_{2}^{2}} M^{\prime}(t)+\int_{0}^{t}\left\|u_{\tau}\right\|_{2}^{2} \mathrm{~d} \tau-\left[J\left(u_{0}\right)+\frac{b(q+1-2 p)}{2(q+1) p}\right] .
\end{aligned}
$$

On the other hand,

$$
\left(M^{\prime}(t)\right)^{2}=4\left(\int_{0}^{t} \int_{\Omega} u_{\tau} u \mathrm{~d} x \mathrm{~d} \tau\right)^{2}+2\left\|u_{0}\right\|_{2}^{2} M^{\prime}(t)-\left\|u_{0}\right\|_{2}^{4} .
$$

Hence, by combining the above two inequalities and applying Cauchy-Schwartz inequality we have

$$
\begin{aligned}
M^{\prime \prime}( & t) M(t)-\frac{q+1}{2} M^{\prime}(t)^{2} \geq 2(q+1) \int_{0}^{t}\left\|u_{\tau}\right\|_{2}^{2} d \tau \int_{0}^{t}\|u\|_{2}^{2} d \tau \\
& -\left(2(q+1) J\left(u_{0}\right)+\frac{b(q+1-2 p)}{p}\right) M(t)+\frac{b(q+1-2 p)}{p S_{2}^{2}} M^{\prime}(t) M(t) \\
& -2(q+1)\left(\int_{0}^{t} \int_{\Omega} u_{\tau} u d x d \tau\right)^{2}-(q+1)\left\|u_{0}\right\|_{2}^{2} M^{\prime}(t)+\frac{q+1}{2}\left\|u_{0}\right\|_{2}^{4} \\
\geq & \frac{b(q+1-2 p)}{p S_{2}^{2}} M^{\prime}(t) M(t)-\left[2(q+1) J\left(u_{0}\right)+\frac{b(q+1-2 p)}{p}\right] M(t) \\
& -(q+1)\left\|u_{0}\right\|_{2}^{2} M^{\prime}(t) .
\end{aligned}
$$


The rest of the proof will be divided into two cases.

(i) $0<J\left(u_{0}\right)<d$. By Lemma 5 we know that $u(t) \in V_{\delta}$ for $t \geq 0$ and $\delta_{1}<\delta<\delta_{2}$, where $\delta_{1}<1<\delta_{2}$ are the two roots of $d(\delta)=J\left(u_{0}\right)$. Hence $I_{\delta_{2}}(u) \leq 0$ and $\|\nabla u\|_{p} \geq r\left(\delta_{2}\right)$ for $t \geq 0$. Then it follows from (3.20) that for $t \geq 0$

$$
\begin{aligned}
& M^{\prime \prime}(t)=-2 I(u)=2 a\left(\delta_{2}-1\right)\|\nabla u\|_{p}^{p}+2 b\left(\delta_{2}-1\right)\|\nabla u\|_{p}^{2 p}-2 I_{\delta_{2}}(u) \\
& \quad \geq 2 a\left(\delta_{2}-1\right) r^{p}\left(\delta_{2}\right),
\end{aligned}
$$

which guarantees that

$$
M^{\prime}(t) \geq 2 a\left(\delta_{2}-1\right) r^{p}\left(\delta_{2}\right) t, \quad M(t) \geq a\left(\delta_{2}-1\right) r^{p}\left(\delta_{2}\right) t^{2} .
$$

Therefore, there exists a $t^{*}>0$ such that for $t \geq t^{*}$, we have

$$
\begin{aligned}
& \frac{b(q+1-2 p)}{2 p S_{2}^{2}} M(t)>(q+1)\left\|u_{0}\right\|_{2}^{2}, \\
& \frac{b(q+1-2 p)}{2 p S_{2}^{2}} M^{\prime}(t)>2(q+1) J\left(u_{0}\right)+\frac{b(q+1-2 p)}{p} .
\end{aligned}
$$

Consequently, from (3.22), we obtain

$$
M^{\prime \prime}(t) M(t)-\frac{q+1}{2} M^{\prime}(t)^{2}>0, \quad t \geq t^{*} .
$$

(ii) $J\left(u_{0}\right) \leq 0$. We will first show that if $J\left(u_{0}\right)<0$ or $J\left(u_{0}\right)=0$ with $\left\|\nabla u_{0}\right\|_{p} \neq 0$, then every solution $u(x, t)$ to problem (1.1) belongs to $V_{\delta}$ for any $0<\delta<\frac{q+1}{2 p}$ and $0 \leq t<T$, where $T>0$ is the maximum existence time. Indeed, from

$$
\frac{\|\nabla u\|_{p}^{p}}{q+1}\left\{\frac{a(q+1-p \delta)}{p}+\frac{b(q+1-2 p \delta)}{2 p}\|\nabla u\|_{p}^{p}\right\}+\frac{I_{\delta}(u)}{q+1}=J(u) \leq J\left(u_{0}\right)
$$

we see that if $J\left(u_{0}\right)<0$, then $J(u(x, t))<0<d(\delta)$ and $I(u(x, t))<0$ for all $0 \leq t<T$, which implies that $u(x, t) \in V_{\delta}$. If $J\left(u_{0}\right)=0$ and $\left\|\nabla u_{0}\right\|_{p} \neq 0$, then $J(u(x, t)) \leq 0$ for all $0 \leq t<T$, which also implies that there exists a constant $c>0$ such that $\|\nabla u(\cdot, t)\|_{p} \geq c$. From (3.23) we again see that $I(u(x, t))<0$ and $J(u(x, t)) \leq 0<d(\delta)$, i.e. $u(x, t) \in V_{\delta}$. Therefore, with $\delta_{2}$ replaced by $\delta$, we can proceed similarly to case (i) to show that (3.23) still holds for sufficiently large $t$.

From (3.23) it follows by Levine's concavity argument (see [12]) that $M(t)$ can not remain finite for all $t>t^{*}$, and therefore reaches a contradiction. The proof is complete.

Remark 1. (Sharp condition for $J\left(u_{0}\right)<d$.) Let $a, b>0, p>\max \{2 n /(n+$ $2), 1\}, 2 p-1<q<p^{*}-1$ and $u_{0} \in W_{0}^{1, p}(\Omega)$. Assume that $J\left(u_{0}\right)<d$. If $I\left(u_{0}\right)>0$, problem (1.1) admits a global weak solution; if $I\left(u_{0}\right)<0$, all solutions to problem (1.1) blow up in finite time. 


\section{$4 \quad \mathbf{J}\left(\mathbf{u}_{0}\right)=\mathbf{d}$}

The invariance of $W$ under the semi-flow of problem (1.1) can not be proved in general for the critical case $J\left(u_{0}\right)=d$. However, by using the method of approximation, we can still obtain the global existence of weak solutions.

Theorem 3. (Global existence for $J\left(u_{0}\right)=$ d.) Assume that $a, b>0, p>$ $\max \{2 n /(n+2), 1\}, 2 p-1<q<p^{*}-1$ and $u_{0} \in W_{0}^{1, p}(\Omega)$. If $J\left(u_{0}\right)=d$ and $I\left(u_{0}\right) \geq 0$, then problem $(1.1)$ admits a global solution $u \in L^{\infty}\left(0, \infty ; W_{0}^{1, p}(\Omega)\right)$ with $u_{t} \in L^{2}\left(0, \infty ; L^{2}(\Omega)\right)$ and $u(t) \in \bar{W}=W \cup \partial W$ for $0 \leq t<\infty$. Moreover, if $I(u(x, t))>0$ for all $t>0$, then there exists a $t_{0}>0$ such that $\|u\|_{2}^{2} \leq$ $\left[\left\|u\left(t_{0}\right)\right\|_{2}^{2-2 p}+C^{*}(p-1)\left(t-t_{0}\right)\right]^{-1 /(p-1)}$, where $C^{*}>0$ is the same constant as that is Theorem 1. If not, then there exists a solution that vanishes in finite time. In addition, the weak solution is unique if it is bounded.

Proof. Let $\lambda_{k}=1-\frac{1}{k}, k=1,2, \ldots$. Consider the following initial boundary value problem

$$
\begin{cases}u_{t}-M\left(\int_{\Omega}|\nabla u|^{p} d x\right) \Delta_{p} u=|u|^{q-1} u, & (x, t) \in \Omega \times(0, T), \\ u=0, & (x, t) \in \partial \Omega \times(0, T), \\ u(x, 0)=\lambda_{k} u_{0}(x) \triangleq u_{0}^{k}, & x \in \Omega .\end{cases}
$$

Since $I\left(u_{0}\right) \geq 0$, it can be deduced from Lemma 2 (iii) that there exists a unique $\lambda^{*}=\lambda^{*}\left(u_{0}\right) \geq 1$ such that $I\left(\lambda^{*} u_{0}\right)=0$. Noticing that $\lambda_{k}<1 \leq \lambda^{*}$, we get $I\left(u_{0}^{k}\right)=I\left(\lambda_{k} u_{0}\right)>0$ and $J\left(u_{0}^{k}\right)=J\left(\lambda_{k} u_{0}\right)<J\left(u_{0}\right)=d$. In view of Theorem 1, it follows that for each $k$ problem (1.1) admits a global weak solution $u^{k} \in L^{\infty}\left(0, \infty ; W_{0}^{1, p}(\Omega)\right)$ with $u_{t}^{k} \in L^{2}\left(0, \infty ; L^{2}(\Omega)\right)$ and $u^{k} \in W$ for $0 \leq t<\infty$ satisfying

$$
\int_{0}^{t}\left\|u_{\tau}^{k}\right\|_{2}^{2} d \tau+J\left(u^{k}\right)=J\left(u_{0}^{k}\right)<d .
$$

Applying similar arguments to those in Theorem 1 we see that there exist a subsequence of $\left\{u^{k}\right\}$ and a function $u$, such that $u$ is a weak solution of problem (1.1) with $I(u) \geq 0$ and $J(u) \leq d$ for $0 \leq t<\infty$. The proof of the uniqueness is the same as that in Theorem 1 .

Let us derive the decay rate of $\|u\|_{2}^{2}$. First, suppose that $I(u)>0$ for $0<t<\infty$, then $u(x, t)$ does not vanish in finite time. Taking $\varphi=u$ in (2.1), we have

$$
\frac{1}{2} \frac{d}{d t}\|u\|_{2}^{2}=\int_{\Omega} u_{t} u d x=-I(u)<0,
$$

which implies that $u_{t} \not \equiv 0$. Therefore, for $t_{0}>0$ suitably small we obtain by (2.2) that

$$
0<J\left(u\left(t_{0}\right)\right)=d-\int_{0}^{t_{0}}\left\|u_{\tau}\right\|_{2}^{2} \mathrm{~d} \tau=d_{1}<d .
$$


Taking $t=t_{0}$ as the initial time and by Lemma 6 (i), we see that $u \in W_{\delta}$ for $\delta_{1}<\delta<\delta_{2}$ and $t>t_{0}$, where $\delta_{1}<1<\delta_{2}$ are the two roots of $d(\delta)=d_{1}$. Hence, $I_{\delta_{1}}(u) \geq 0$ for $t>t_{0}$ and

$$
\begin{aligned}
\frac{1}{2} \frac{d}{d t}\|u\|_{2}^{2} & =-I(u)=a\left(\delta_{1}-1\right)\|\nabla u\|_{p}^{p}+b\left(\delta_{1}-1\right)\|\nabla u\|_{p}^{2 p}-I_{\delta_{1}}(u) \\
& \leq \frac{b\left(\delta_{1}-1\right)}{S_{2}^{2 p}}\|u\|_{2}^{2 p}
\end{aligned}
$$

Integrating the above inequality over $\left[t_{0}, t\right]$ for any $t>t_{0}$ yields

$$
\|u\|_{2}^{2} \leq\left[\left\|u\left(t_{0}\right)\right\|_{2}^{2-2 p}+C^{*}(p-1)\left(t-t_{0}\right)\right]^{-1 /(p-1)} .
$$

Next, suppose that $I(u)>0$ for $0<t<t_{*}$ and $I\left(u\left(x, t_{*}\right)\right)=0$. Obviously, $u_{t} \not \equiv 0$ for $0<t<t_{*}$ and $\int_{0}^{t_{*}}\left\|u_{\tau}\right\|_{2}^{2} \mathrm{~d} \tau>0$. Applying (2.2) again, we have

$$
J\left(u\left(t_{*}\right)\right)=d-\int_{0}^{t_{*}}\left\|u_{\tau}\right\|_{2}^{2} \mathrm{~d} \tau=d_{1}<d .
$$

By the variational formula of $d$, we know $\left\|\nabla u\left(t_{*}\right)\right\|_{p}=0$, which implies $u\left(t_{*}\right)=$ 0 . Define $u(x, t) \equiv 0$ for all $t \geq t_{*}$. Then it is seen that such a $u(x, t)$ is a weak solution of (1.1) that vanishes in finite time. The proof is complete.

Theorem 4. (Blow-up for $J\left(u_{0}\right)=$ d.) Assume $a, b>0, p>\max \{2 n /(n+$ $2), 1\}, 2 p-1<q<p^{*}-1$ and $u_{0} \in W_{0}^{1, p}(\Omega)$. If $J\left(u_{0}\right)=d$ and $I\left(u_{0}\right)<0$, then there exists a finite time $T$ such that $u$ blows up at $T$ in the sense that $\lim _{t \rightarrow T} \int_{0}^{t}\|u\|_{2}^{2} \mathrm{~d} \tau=+\infty$.

Proof. Similarly to the proof of Theorem 2, we can get

$$
\begin{aligned}
& M^{\prime \prime}(t) M(t)-\frac{q+1}{2} M^{\prime}(t)^{2} \geq\left\{\frac{b(q+1-2 p)}{2 p S_{2}^{2}} M^{\prime}(t)\right. \\
& \left.-\left(2(q+1) d+\frac{b(q+1-2 p)}{p}\right)\right\} M(t) \\
& \quad+\left\{\frac{b(q+1-2 p)}{2 p S_{2}^{2}} M(t)-(q+1)\left\|u_{0}\right\|_{2}^{2}\right\} M^{\prime}(t) .
\end{aligned}
$$

Since $J\left(u_{0}\right)=d, I\left(u_{0}\right)<0$, by the continuity of $J(u)$ and $I(u)$ with respect to $t$, there exists a $t^{0}>0$ such that $J(u(x, t))>0$ and $I(u(x, t))<0$ for $0<t \leq t^{0}$. From $\left(u_{t}, u\right)=-I(u)$, it is known that $u_{t} \neq \equiv 0$ for $0<t \leq t^{0}$. Furthermore, we have

$$
J\left(u\left(t^{0}\right)\right)=d-\int_{0}^{t^{0}}\left\|u_{\tau}\right\|_{2}^{2} \mathrm{~d} \tau=d_{1}<d .
$$

Taking $t=t^{0}$ as the initial time and by Lemma 6 (ii), we know that $u(x, t) \in V_{\delta}$ for $\delta_{1}<\delta<\delta_{2}$ and $t>t^{0}$, where $\delta_{1}<1<\delta_{2}$ are the two roots of the equation 
$d(\delta)=d_{1}$. Therefore, we have $I_{\delta}(u)<0$ and $\|\nabla u\|_{p}>r(\delta)$ for $\delta_{1}<\delta<\delta_{2}$ and $t>t^{0}$. Thus, $I_{\delta_{2}}(u) \leq 0$ and $\|\nabla u\|_{p} \geq r\left(\delta_{2}\right)$ for $t>t^{0}$. Then for $t>t^{0}$ we get the following estimates

$$
\begin{aligned}
& M^{\prime \prime}(t)=-2 I(u)=2 a\left(\delta_{2}-1\right)\|\nabla u\|_{p}^{p}+2 b\left(\delta_{2}-1\right)\|\nabla u\|_{p}^{2 p}-2 I_{\delta_{2}}(u) \\
& \quad \geq 2 a\left(\delta_{2}-1\right) r^{p}\left(\delta_{2}\right), \\
& M^{\prime}(t) \geq 2 a\left(\delta_{2}-1\right) r^{p}\left(\delta_{2}\right)\left(t-t^{0}\right), \quad M(t) \geq a\left(\delta_{2}-1\right) r^{p}\left(\delta_{2}\right)\left(t-t^{0}\right)^{2} .
\end{aligned}
$$

Consequently, for sufficiently large $t$, we get from (4.1) that

$$
M^{\prime \prime}(t) M(t)-\frac{q+1}{2} M^{\prime}(t)^{2}>0 .
$$

The rest of the proof is the same as that of Theorem 2 and hence is omitted.

Remark 2. (Sharp condition for $J\left(u_{0}\right)=d$.) Let $a, b>0, p>\max \{2 n /(n+$ 2), 1$\}, 2 p-1<q<p^{*}-1$ and $u_{0} \in W_{0}^{1, p}(\Omega)$. Assume that $J\left(u_{0}\right)=d$. If $I\left(u_{0}\right) \geq 0$, problem (1.1) admits a global weak solution; if $I\left(u_{0}\right)<0$, all solutions to problem (1.1) blow up in finite time.

\section{$5 \quad J\left(u_{0}\right)>d$}

Inspired by some ideas from $[7,10,24]$, we can give, in terms of $\lambda_{s}$ and $\Lambda_{s}$, an abstract criterion for the existence of global solutions that vanish at infinity or solutions that blow up in finite time, when the initial energy is lager than the depth of the potential well, i.e. $J\left(u_{0}\right)>d$. To do this, we denote by $T\left(u_{0}\right)$ the maximal existence time of the solutions to problem (1.1) with initial datum $u_{0}$. For fixed time $t \in\left[0, T\left(u_{0}\right)\right)$, we think of the function $u(x, t)$ of the space variable $x$ as an element of $W_{0}^{1, p}(\Omega)$, and briefly denote the element of $W_{0}^{1, p}(\Omega)$ that arises this way by $u(t)$; therefore $u(t) \in W_{0}^{1, p}(\Omega)$. If now we vary the time $t$ in the interval $\left[0, T\left(u_{0}\right)\right)$, then we obtain a function $t \rightarrow u(t)$. If the solution is global, i.e. $T\left(u_{0}\right)=\infty$, we denote by

$$
\omega\left(u_{0}\right)=\bigcap_{t \geq 0} \overline{\{u(s): s \geq t\}} W_{0}^{1, p}(\Omega)
$$

the $\omega$-limit set of $u_{0}$.

Theorem 5. Assume $2 p<q+1<p^{*}$. If $J\left(u_{0}\right)>d$, then the following statements hold

(i) If $u_{0} \in \mathcal{N}_{+}$and $\left\|u_{0}\right\|_{2} \leq \lambda_{J\left(u_{0}\right)}$, then $u_{0} \in \mathcal{G}_{0}$;

(ii) If $u_{0} \in \mathcal{N}_{-}$and $\left\|u_{0}\right\|_{2} \geq \Lambda_{J\left(u_{0}\right)}$, then $u_{0} \in \mathcal{B}$.

Proof. (i) Suppose that $u_{0} \in \mathcal{N}_{+}$satisfying $\left\|u_{0}\right\|_{2} \leq \lambda_{J\left(u_{0}\right)}$. We first claim that $u(t) \in \mathcal{N}_{+}$for all $t \in\left[0, T\left(u_{0}\right)\right)$. If not, there would exist a $t_{0} \in\left(0, T\left(u_{0}\right)\right)$ such that $u(t) \in \mathcal{N}_{+}$for $0 \leq t<t_{0}$ and $u\left(t_{0}\right) \in \mathcal{N}$. On the other hand, it 
follows from (2.4) that $J\left(u\left(t_{0}\right)\right) \leq J\left(u_{0}\right)$, which means that $u\left(t_{0}\right) \in J^{J\left(u_{0}\right)}$. Therefore, $u\left(t_{0}\right) \in \mathcal{N}^{J\left(u_{0}\right)}$. According to the definition of $\lambda_{J\left(u_{0}\right)}$, we have

$$
\left\|u\left(t_{0}\right)\right\|_{2} \geq \lambda_{J\left(u_{0}\right)} .
$$

Taking $\phi=u$ in (2.3), we get

$$
\frac{1}{2} \frac{d}{d t}\|u\|_{2}^{2}=\left(u_{t}, u\right)=-a\|\nabla u\|_{p}^{p}-b\|\nabla u\|_{p}^{2 p}+\|u\|_{q+1}^{q+1}=-I(u) .
$$

Since $I(u(t))>0$ for $t \in\left[0, t_{0}\right),(5.2)$ ensures that

$$
\left\|u\left(t_{0}\right)\right\|_{2}<\left\|u_{0}\right\|_{2} \leq \lambda_{J\left(u_{0}\right)},
$$

which is contradictive with (5.1). So $u(t) \in \mathcal{N}_{+}$, as claimed.

Recalling Lemma 7 (ii), one can see that the orbit $\{u(t)\}$ is bounded in $W_{0}^{1, p}(\Omega)$ for $t \in\left[0, T\left(u_{0}\right)\right)$ so that $T\left(u_{0}\right)=\infty$. Let $\omega$ be an arbitrary element in $\omega\left(u_{0}\right)$, then by $(2.4)$ and $(5.2)$ we have

$$
\|\omega\|_{2}<\lambda_{J\left(u_{0}\right)}, \quad J(\omega) \leq J\left(u_{0}\right) .
$$

The above inequality, together with the definition of $\lambda_{J\left(u_{0}\right)}$, implies $\omega\left(u_{0}\right) \cap \mathcal{N}=$ $\emptyset$. Therefore, $\omega\left(u_{0}\right)=\{0\}$, i.e. $u_{0} \in \mathcal{G}_{0}$.

(ii) Assume that $u_{0} \in \mathcal{N}_{-}$with $\left\|u_{0}\right\|_{2} \geq \Lambda_{J\left(u_{0}\right)}$. By applying similar argument as above we see that $u(t) \in \mathcal{N}_{-}$for all $t \in\left[0, T\left(u_{0}\right)\right)$. Now if $T\left(u_{0}\right)=\infty$, then for every $\omega \in \omega\left(u_{0}\right)$, it follows again from (2.4) and (5.2) that

$$
\|\omega\|_{2}>\Lambda_{J\left(u_{0}\right)}, \quad J(\omega) \leq J\left(u_{0}\right) .
$$

Combining (5.3) with the definition of $\Lambda_{J\left(u_{0}\right)}$, we see that $\omega\left(u_{0}\right) \cap \mathcal{N}=\emptyset$. Thus, it must hold that $\omega\left(u_{0}\right)=\{0\}$, which is contradictive with Lemma 7 (i). Therefore, $T\left(u_{0}\right)<\infty$ and $u_{0} \in \mathcal{B}$. The proof is complete.

Theorem 5 (ii) implies that for any $M>d$, there exists a $u_{0}$ such that $J\left(u_{0}\right)>M$ and that the solutions to problem (1.1) with $u_{0}$ as initial datum blow up in finite time. This is illustrated in the following two corollaries.

Corollary 1. Let $2 p<q+1<p^{*}$ and $u_{0} \in W_{0}^{1, p}(\Omega)$ such that $J\left(u_{0}\right)>d$. If $\frac{2 p(q+1)}{q+1-2 p}|\Omega|^{(q-1) / 2} J\left(u_{0}\right) \leq\left\|u_{0}\right\|_{2}^{q+1}$, then $u_{0} \in \mathcal{N}_{-} \cap \mathcal{B}$.

Proof. It follows from

$$
\frac{2 p(q+1)}{q+1-2 p}|\Omega|^{(q-1) / 2} J\left(u_{0}\right) \leq\left\|u_{0}\right\|_{2}^{q+1}
$$

and Hölder's inequality that

$$
\frac{2 p(q+1)}{q+1-2 p}|\Omega|^{(q-1) / 2} J\left(u_{0}\right) \leq\left\|u_{0}\right\|_{2}^{q+1}<\left\|u_{0}\right\|_{q+1}^{q+1}|\Omega|^{(q-1) / 2} .
$$


The last inequality is strict because $u_{0}$ is not a constant in $\Omega$. By combining the expression of $J\left(u_{0}\right), I\left(u_{0}\right)$ with $(5.4)$ we have

$$
\begin{aligned}
J\left(u_{0}\right) & =\frac{a}{p}\left\|\nabla u_{0}\right\|_{p}^{p}+\frac{b}{2 p}\left\|\nabla u_{0}\right\|_{p}^{2 p}-\frac{1}{q+1}\left\|u_{0}\right\|_{q+1}^{q+1}, \\
& =\frac{a}{2 p}\left\|\nabla u_{0}\right\|_{p}^{p}+\left(\frac{1}{2 p}-\frac{1}{q+1}\right)\left\|u_{0}\right\|_{q+1}^{q+1}+\frac{1}{2 p} I\left(u_{0}\right) \\
& >\frac{q+1-2 p}{2 p(q+1)}\left\|u_{0}\right\|_{q+1}^{q+1}+\frac{1}{2 p} I\left(u_{0}\right)>J\left(u_{0}\right)+\frac{1}{2 p} I\left(u_{0}\right),
\end{aligned}
$$

which shows that $I\left(u_{0}\right)<0$, i.e. $u_{0} \in \mathcal{N}_{-}$.

In order to show that $u_{0} \in \mathcal{B}$, it remains to prove $\left\|u_{0}\right\|_{2} \geq \Lambda_{J\left(u_{0}\right)}$ by Theorem 5 (ii). For this, $\forall u \in \mathcal{N}^{J\left(u_{0}\right)}$, we have

$$
\begin{aligned}
\|u\|_{2}^{q+1} & \leq|\Omega|^{(q-1) / 2}\|u\|_{q+1}^{q+1}=|\Omega|^{(q-1) / 2}\left(a\|\nabla u\|_{p}^{p}+b\|\nabla u\|_{p}^{2 p}\right) \\
& =|\Omega|^{(q-1) / 2} \frac{2 p(q+1)}{q+1-2 p}\left\{\left(\frac{1}{2 p}-\frac{1}{q+1}\right) a\|\nabla u\|_{p}^{p}+\left(\frac{1}{2 p}-\frac{1}{q+1}\right) b\|\nabla u\|_{p}^{2 p}\right\} \\
& <|\Omega|^{(q-1) / 2} \frac{2 p(q+1)}{q+1-2 p}\left\{\left(\frac{1}{p}-\frac{1}{q+1}\right) a\|\nabla u\|_{p}^{p}+\left(\frac{1}{2 p}-\frac{1}{q+1}\right) b\|\nabla u\|_{p}^{2 p}\right\} \\
& =|\Omega|^{(q-1) / 2} \frac{2 p(q+1)}{q+1-2 p} J\left(u_{0}\right) \leq\left\|u_{0}\right\|_{2}^{q+1} .
\end{aligned}
$$

Taking supremum over $\mathcal{N}^{J\left(u_{0}\right)}$ we obtain

$$
\Lambda_{J\left(u_{0}\right)}^{q+1} \leq|\Omega|^{(q-1) / 2} \frac{2 p(q+1)}{q+1-2 p} J\left(u_{0}\right) \leq\left\|u_{0}\right\|_{2}^{q+1},
$$

i.e. $\left\|u_{0}\right\|_{2} \geq \Lambda_{J\left(u_{0}\right)}$. Therefore, $u_{0} \in \mathcal{N}_{-} \cap \mathcal{B}$. The proof is complete.

Corollary 2. For any $M>d$, there exists a $u_{M} \in \mathcal{N}_{-}$such that $J\left(u_{M}\right) \geq M$ and $u_{M} \in \mathcal{B}$.

Proof. Similar treatments have been used in $[7,9,24]$ to deal with semilinear parabolic problem and pseudo-parabolic problem, respectively. We repeat the proof here for the convenience of the readers. For any $M>d$, let $\Omega_{1}$ and $\Omega_{2}$ be two arbitrary disjoint open subdomains of $\Omega$, and assume that $v \in W_{0}^{1, p}\left(\Omega_{1}\right)$ is an arbitrary nontrivial function. Since $q+1>2 p$, we can choose $\alpha>0$ large enough such that $J(\alpha v) \leq 0$ and $\|\alpha v\|_{2}^{q+1} \geq|\Omega|^{(q-1) / 2} \frac{2 p(q+1)}{q+1-2 p} M$.

Fix such an $\alpha$ and then choose a function $w \in W_{0}^{1, p}\left(\Omega_{2}\right)$ such that $J(w)+$ $J(\alpha v)=M$. Extend $v$ and $w$ to be 0 in $\Omega \backslash \Omega_{1}$ and $\Omega \backslash \Omega_{2}$, respectively, and set $u_{M}=\alpha v+w$. Then $J\left(u_{M}\right)=J(\alpha v)+J(w)=M$ and

$$
\left\|u_{M}\right\|_{2}^{q+1} \geq\|\alpha v\|_{2}^{q+1} \geq|\Omega|^{(q-1) / 2} \frac{2 p(q+1)}{q+1-2 p} J\left(u_{M}\right) .
$$

By Corollary 1 it is seen that $u_{M} \in N_{-} \cap \mathcal{B}$. The proof is complete. 


\section{Acknowledgements}

The authors would like to thank the referees for their valuable comments and suggestions which improve the original manuscript. Jian Li is supported by NSFC (No. 41601454). Yuzhu Han is supported by NSFC (No. 11401252), by The Education Department of Jilin Province (JJKH20190018KJ) and by Science and Technology Development Plan Project of Jilin Province (20160520103JH). The authors would also like to express their sincere gratitude to Professor Wenjie Gao for his enthusiastic guidance and constant encouragement.

\section{References}

[1] H. Brezis. Functional Analysis, Sobolev Spaces and Partial Differential Equations. Springer, New York, 2010.

[2] M. Chipot and T. Savitska. Nonlocal $p$-Laplace equations depending on the $L^{p}$ norm of the gradient. Adv. Differential Equations, 19(11-12):997-1020, 2014.

[3] M. Chipot, V. Valente and G. V. Caffarelli. Remarks on a nonlocal problems involving the Dirichlet energy. Rend. Sem. Math. Univ. Padova, 110(4):199-220, 2003 .

[4] P. D'Ancona and Y. Shibata. On global solvability of non-linear viscoelastic equation in the analytic category. Math. Methods Appl. Sci., 17(6):477-489, 1994. https://doi.org/10.1002/mma.1670170605.

[5] P. D'Ancona and S. Spagnolo. Global solvability for the degenerate Kirchhoff equation with real analytic data. Invent. Math., 108(1):247-262, 1992. https://doi.org/10.1007/BF02100605.

[6] Y.Q. Fu and M.Q. Xiang. Existence of solutions for parabolic equations of Kirchhoff type involving variable exponent. Appl. Anal., 95(3):524-544, 2016. https://doi.org/10.1080/00036811.2015.1022153.

[7] F. Gazzola and T. Weth. Finite time blow up and global solutions for semilinear parabolic equations with initial data at high energy level. Differential Integral Equations, 18(9):961-990, 2005.

[8] M. Ghisi and M. Gobbino. Hyperbolic-parabolic singular perturbation for mildly degenerate Kirchhoff equations: time-decay estimates. J. Differential Equations, 245(10):2979-3007, 2008. https://doi.org/10.1016/j.jde.2008.04.017.

[9] Y.Z. Han. A class of fourth-order parabolic equation with arbitrary initial energy. Nonlinear Anal. Real World Appl., 43:451-466, 2018. https://doi.org/10.1016/j.nonrwa.2018.03.009.

[10] Y.Z. Han and Q.W. Li. Threshold results for the existence of global and blow-up solutions to Kirchhoff equations with arbitrary initial energy. Computers Math. Appl., 75(9):3283-3297, 2018. https://doi.org/10.1016/j.camwa.2018.01.047.

[11] G. Kirchhoff. Mechanik. Teubner, Leipzig, 1883.

[12] H.A. Levine. Some nonexistence and instability theorems for solutions of formally parabolic equation of the form $p u_{t}=-a u+\mathcal{F} u$. Arch. Rati. Mech. Anal., 51(5):371-386, 1973. https://doi.org/10.1007/BF00263041. 
[13] Q.W. Li, W.J. Gao and Y.Z. Han. Global existence blow up and extinction for a class of thin-film equation. Nonlinear Anal., 147:96-109, 2016. https://doi.org/10.1016/j.na.2016.08.021.

[14] J.L. Lions. Quelques methods de resolution des problem aux limits nonlinears. Dunod, Paris, 1969.

[15] J.L. Lions. On some questions in boundary value problems of mathematical physics. North-Holland Mathematical Studies, 30:284-346, 1978. https://doi.org/10.1016/S0304-0208(08)70870-3.

[16] Y.C. Liu. On potential wells and vacuum isolating of solutions for semilinear wave equations. J. Differential Equations, 192(1):155-169, 2003. https://doi.org/10.1016/S0022-0396(02)00020-7.

[17] K. Nishihara. On a global solution of some quasilinear hyperbolic equation. Tokyo J. Math., 7(2):437-459, 1984. https://doi.org/10.3836/tjm/1270151737.

[18] N. Pan, B. L. Zhang and J. Cao. Degenerate Kirchhoff-type diffusion problems involving the fractional p-Laplacian. Nonlinear Anal. Real World Appl., 37:5670, 2017. https://doi.org/10.1016/j.nonrwa.2017.02.004.

[19] L.E. Payne and D.H. Sattinger. Saddle points and instability of nonlinear hyperbolic equtions. Israel J. Math., 22(3-4):273-303, 1975. https://doi.org/10.1007/BF02761595.

[20] P. Pucci, M. Q. Xiang and B. L. Zhang. A diffusion problem of Kirchhoff type involving the nonlocal fractional $p$-Laplacian. Discrete Contin. Dyn. Syst., 37:4035-4051, 2017. https://doi.org/10.3934/dcds.2017171.

[21] C.Y. Qu and W.S. Zhou. Blow-up and extinction for a thin-film equation with initial-boundary value conditions. J. Math. Anal. Appl., 436(2):796-809, 2016. https://doi.org/10.1016/j.jmaa.2015.11.075.

[22] D.H. Sattinger. On global solution of nonlinear hyperbolic equations. Arch. Rati. Mech. Anal., 30(2):148-172, 1968. https://doi.org/10.1007/BF00250942.

[23] M. Q. Xiang, V. D. Rădulescu and B. L. Zhang. Nonlocal Kirchhoff diffusion problems: local existence and blow-up of solutions. Nonlinearity, 31(7):32283250, 2018. https://doi.org/10.1088/1361-6544/aaba35.

[24] R.Z. Xu and J. Su. Global existence and finite time blow-up for a class of semilinear pseudo-parabolic equations. J. Funct. Anal., 264(12):2732-2763, 2013. https://doi.org/10.1016/j.jfa.2013.03.010. 